\title{
A Case of Multiple System Atrophy (MSA) - First Presentation in a Psychiatry Service
}

Elena Alina ROSCA ${ }^{\top}$, Andra Livia BRONESCU ${ }^{1}$, Ovidiu Iulian TUDOR ${ }^{1}$

\section{Abstract}

A 67 years old female developed cognitive defficits, depression and anxiety as first symptoms of multiple system atrophy (MSA). In the course of an year autonomic failure, parkinsonism and cerebellar ataxia also developed. The case is particular because of the somatic symptoms that were initially categorised as psychogenic, the presence of confusional and vertigo episodes that were recurring but not permanent.

Keywords: cognitive defficits, depression, anxiety, multiple system atrophy, psychiatry.

${ }^{1}$ "Carol Davila" University of Medicine and Pharmacy, Bucharest, Romania
Corresponding author:

Elena Alina ROSCA, "Carol Davila" University of Medicine and Pharmacy, Bucharest, Romania. 


\section{INTRODUCTION}

Multiple system atrophy (MSA) is a rare progressive neurodegenerative disease with adult onset caused by cell loss, gliosis, glial cytoplasmic inclusions in striatonigral olivopontocerebellar and autonomic systems. It is characterized clinically by parkinsonism, cerebellar ataxia, autonomic failure and corticospinal signs with poor or no response to levodopa ${ }^{1}$. Female gender, longer disease duration, and more serious disease severity represent common predictors for depression and anxiety found in MSA ${ }^{2}$.

\section{CASE PRESENTATION}

We present a case of a female patient (67 years old) without psychiatric history, who was observed over the course of 6 years (2015-2021). She reported no alcohol, tobacco or drug abuse and no family history of psychiatric disorders.

September 2015: Patient was addmitted to our hospital for depressed mood, mild attention and memory defficits, difficulties maintaing sleep, fluctuanting appetite, paresthesia, fatigue and reccurent episodes of tremor, sweating, dizzines, nausea and vomiting.

Blood analysis was performed with the following results: blood count and leukocite formula in normal ranges, normal thyroid function, glucose in normal range, normal ionogram, liver and renal function normal except a mild increase of TGP (39 U/L) and no inflammatory markers identified. Abdominal ultrasound showed postcholecystectomy dilatation of the bile duct.

MRI scan showed milimetrical white matter hyperintensity lesions on $\mathrm{T} 2$ and fluid attenuated inversion recovery (FLAIR) in fronto-parietal cortex (demielinizations, gliosis), no diffusion restrictions, mild supratentorial atrophy more emphasized in bilateral fronto-parietal cortex with secundary deepening of fronto-parietal sulci and interemispherical fissure, deepening of cereberal sulci. MRI results were interpreted as most probably having a microangiopathic cause. EEG was normal.

Patient was diagnosed with moderate depressive episode, mild cognitive impairment and unspecified poyneuropathy and was treated with a 10 day-cure of intravenous nootropic agent Cerebrolysin and a longterm treatment with tianeptine $37,5 \mathrm{mg} /$ day and gabapentine $600 \mathrm{mg} /$ day was initiated.
December 2015: Patient returned for the persistence of neurocognitive deficits and depressed mood and new symptoms: increased anxiety, iritability, anhedonia, thought of hopelessness and worsening of the neurovegetative and gastrointestinal symptoms. She reported partial compliance to the psychiatric treatment. We decided to reinitiate the same psychiatric treatment together with low doses of lorazepam (1-2 mg/day) and continue investigating possible somatic comorbidities.

February 2016: Patient was admitted for follow-up. Depressive symptomatology worsened, patient showed marked psycho-motor inhibition with sad facies, slow movements and hypophonia. Pathological crying was reported: "I have frequent episodes that last a few seconds when I feel my head going soft, dizziness, nausea, I start to cry and shake.". Head CT scan showed moderate cerebral and cerebellar atrophy. We maintaned the nootropic treatment and decided to switch the antidepressant medication to Escitalopram $10 \mathrm{mg} /$ day.

March 2016: Patient underwent an internal medicine examination after 10 days of digestive intolerance, nausea, vomiting and epigastric pain. Blood tests excluded liver or pancreatic disease, blood count and inogram were normal. Abdominal CT scan: Postcholecystectomy syndrome. Upper endoscopy: Erythematous gastritis. Final diagnosis was psychogenic vomiting.

April 2016: Recurring episodes of dizziness and pathological crying became more frequent and intense and associated confusion. Vertigo was more intense in orthostatic position and during left-right head movements. Diagnosis: Recurring nonspecified vestibular disorder.

June 2016: Patient was admitted for follow-up. Psychological tests showed: $\mathrm{MMSE}=22 / 30, \mathrm{ADL}=6 / 6$, IADL $=6 / 8$, GDS Reisberg stage $=4, \mathrm{~W}-\mathrm{M}$ questionaire with pathological scores for depression, anxiety, hipochondria and instability. Confusional and vertigo episodes worsened.

Blood pressure was $110 / 60 \mathrm{mmHg}$ in clinostatism and $80 / 50 \mathrm{mmHg}$ in orthostatism. Ambulatory blood pressure test results: mean diurnal $\mathrm{BP}=102 / 61$ $\mathrm{mmHg}$, mean nocturnal $\mathrm{BP}=82 / 46 \mathrm{mmHg}$. Cervical Doppler ultrasound: carotidian atheromatosis with no hemodinamic consequences. Neurological examination showed: no coordination disorder, no motor or sensitivity deficits, positive Romberg test, bilateral hand tremor, positive bilateral Noica sign, normal ostheo-tendinous reflexes, ubnormal posture reflexes. Diagnosis: Parkinson-like syndrome in observation. Orthostatic 
and nocturnal hypotension. Carotidian atheromatosis.

Patient showed no response to Escitalopram therefore we switched to Venlafaxine $37.5 \mathrm{mg} /$ day. Treatment with Midodrinum $5 \mathrm{mg} /$ day and Vincaminum $60 \mathrm{mg} /$ day was initiated.

September 2016: All medical data were gathered and the diagnosis of multiple system atophy was formulated. Levodopum $250 \mathrm{mg} /$ Carbidopum $25 \mathrm{mg}$ and Risperidone $0.5 \mathrm{mg}$ were added to the patient treatment.

March 2020: Patient returned for follow-up after 3,5 years of slowly progressive disease and showed: no ability to walk, FMS scale $4 / 5$ superior loins, $2 / 5$ inferior loins, $\mathrm{ADL}=2 / 6, \mathrm{IADL}=0 / 8$, urinary and $\mathrm{fe}-$ cal incontinence, dysmetria. Psychiatric examination showed temporal and spatial disorientation, severe attention and memory defficits (MMSE=9/30), bradypsyhia, bradylalia, depressed mood, visual and auditive hallucinations, episodes of psycho-motor agitation., GAFS=10/100. Diagnosis of dementia was formulated and treatment with Rivastigmine was initiated. The disease progressed to exitus by the begining of 2021 .

\section{References}

1. Gilman S, Wenning GK, Low PA, Brooks DJ, Mathias CJ, Trojanowski JQ, Wood NW, Co- losimo C, Dürr A, Fowler CJ, Kaufmann H, Klockgether T, Lees A, Poewe W, Quinn N, Revesz T, Robertson D, Sandroni P, Seppi K, Vidailhet M: Second consensus statement on the diagnosis of multiple system atrophy. Neurology 2008;71:670-676.

2. Zhang, L.-Y., Cao, B., Zou, Y.-T., Wei, Q.-Q., Ou, R.-W., Zhao, B., ... Shang, H.-F. (2017). Depression and anxiety in multiple system atrophy. Acta Neurologica Scandinavica, 137(1), 3337. doi:10.1111/ane. 12804 